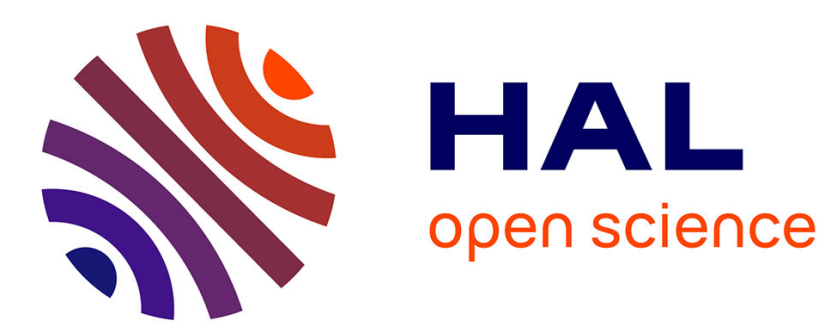

\title{
Strain localization analysis using a multiscale model
}

Gérald Franz, Farid Abed-Meraim, Tarak Ben Zineb, Xavier Lemoine, Marcel Berveiller

\section{To cite this version:}

Gérald Franz, Farid Abed-Meraim, Tarak Ben Zineb, Xavier Lemoine, Marcel Berveiller. Strain localization analysis using a multiscale model. Computational Materials Science, 2009, 45 (3), pp.768773. 10.1016/j.commatsci.2008.05.033 . hal-01084136

\section{HAL Id: hal-01084136 https://hal.science/hal-01084136}

Submitted on 18 Nov 2014

HAL is a multi-disciplinary open access archive for the deposit and dissemination of scientific research documents, whether they are published or not. The documents may come from teaching and research institutions in France or abroad, or from public or private research centers.
L'archive ouverte pluridisciplinaire HAL, est destinée au dépôt et à la diffusion de documents scientifiques de niveau recherche, publiés ou non, émanant des établissements d'enseignement et de recherche français ou étrangers, des laboratoires publics ou privés. 


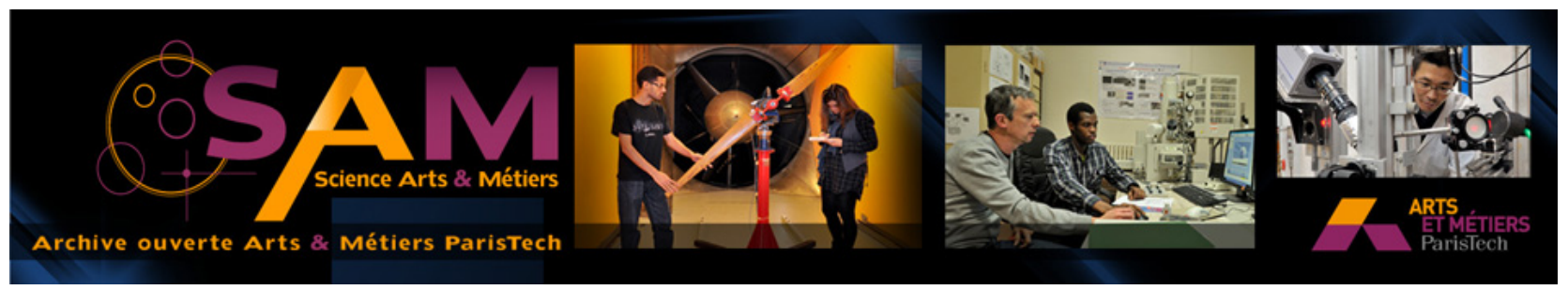

Science Arts \& Métiers (SAM)

is an open access repository that collects the work of Arts et Métiers ParisTech researchers and makes it freely available over the web where possible.

This is an author-deposited version published in: http://sam.ensam.eu

Handle ID: .http://hdl.handle.net/10985/8935

\section{To cite this version :}

Gérald FRANZ, Farid ABED-MERAIM, Tarak BEN ZINEB, Xavier LEMOINE, Marcel BERVEILLER - Strain localization analysis using a multiscale model - Computational materials science - Vol. 45, n`3, p.768-773 - 2009 


\title{
Strain localization analysis using a multiscale model
}

\author{
G. Franz ${ }^{a}$, F. Abed-Meraim ${ }^{a,},{ }^{*}$ T. Ben Zineb ${ }^{b}$, X. Lemoine ${ }^{c}$, M. Berveiller ${ }^{a}$ \\ ${ }^{a}$ LPMM, UMR CNRS 7554, ENSAM de Metz, 4 rue Augustin Fresnel, 57078 Metz Cedex 3, France \\ ${ }^{b}$ LEMTA, UMR CNRS 7563, Nancy University, 2 rue Jean Lamour, 54519 Vandoeuvre-lès-Nancy, France \\ ${ }^{c}$ R\&D Automotive Products, ArcelorMittal Maizières, voie Romaine, B.P. 30320, 57283 Maizières-lès-Metz, France
}

\begin{abstract}
In order to analyze the formability of steels in sheet metal forming, a ductility loss criterion is coupled with a multiscale model. The behavior at the mesoscopic (grain) scale is modeled by a large strain micromechanical constitutive law, which is then used in a self-consistent scale transition scheme. Hardening at the slip system level is taken into account through mean dislocation densities considered as internal variables. The determination of active slip systems and the calculation of plastic slip activity are achieved with help of a regularization technique drawn from viscoplastic formulations. The model is shown to be able to correctly simulate the macroscopic behavior for single-phase steels during both monotonic and sequential loading paths. Finally, Rice's localization criterion, based on the ellipticity loss of the elastic-plastic tangent modulus, is introduced and applied to determine Forming Limit Diagrams (FLDs). The model allows us to obtain correct FLDs for monotonic as well as sequential loading paths. Pre-strain impact on FLDs is qualitatively reproduced as well.
\end{abstract}

Keywords: Crystal plasticity; Scale transition; Ductility; Forming Limit Diagram; Bifurcation; Complex loading paths PACS: 61.72.Ff; 61.72.-y; 62.20.Fe; 83.10.Gr; 83.60.-a

\section{Introduction}

The concept of Forming Limit Diagrams (FLDs) was first introduced by Keeler [1] and Goodwin [2] in order to predict critical strains leading to material failure for different strain paths. It has long been used to assess the formability of stretched sheet metals. Since the onset of localized necking is nowadays the main limitation of industrial forming processes, the FLDs are commonly plotted at localization.

During sheet metal forming processes, several failure modes may occur (buckling, wrinkling, diffuse and localized necking) and can sometimes even be coupled with damage phenomena. As a unified approach taking into account all of the abovementioned mechanisms seems to be very difficult, the present work only focuses on the onset of strain localization due to macroscopic shear band formation.

In order to predict this phenomenon, two main approaches are usually adopted. The MarciniakKuczynski (M-K) model [3] and its generalization developed by Hutchinson and Neale [4] assume the existence of an initial imperfection materialized by an oriented band through the material sheet. This band represents a locally weak region of the specimen. The difference in thickness between the two regions grows during loading and the ductility limit is attained when this difference reaches a critical value. This approach is widely used to simulate FLDs and the obtained results are usually in agreement with experimental data. However, the main drawback of this theory is the high sensitivity of the results to some parameters such as the initial defect thickness or the critical value.
The second approach, introduced by Rudnicki-Rice $[5,6]$, is based on the formation of strain localization bands corresponding to jumps of mechanical fields across interfaces. This theory is related to the ellipticity loss of the elastic-plastic tangent modulus. The condition of localization is reached when the first zero eigenvalue is seen in the acoustic tensor. Since this approach, called discontinuous bifurcation theory, does not need any additional parameters and can be applied to three-dimensional problems, it is preferred to the classical $\mathrm{M}-\mathrm{K}$ approach for this current work.

The main objective here is to predict the ductility loss with maximum accuracy by taking into account the influence of changing loading paths and mechanical properties with the aim of designing new materials. For this purpose, Rice's criterion will be coupled with a constitutive law derived from a large strain micromechanical approach and a self-consistent scale transition scheme. Comparisons with experiments, for several sequential rheological tests, are presented for a single-phase steel. The ductility limit predictions obtained by Rice's criterion for this steel are then discussed using the concept of FLDs.

\section{Single crystal constitutive law}

According to the pioneering contributions [7-10], the elastic-plastic single crystal constitutive law modeled within the large strain framework is presented here. Several assumptions are adopted for the material behavior modeling at the mesoscopic scale. The single crystal behavior is assumed elasticplastic and the plastic deformation is considered to be

\footnotetext{
" Corresponding author. Tel.: +33 3 87375479; Fax: +33 387374284.
}

E-mail address: farid.abed-meraim@metz.ensam.fr 
only due to crystallographic slip; the other plastic strain modes like twinning or phase transformation are not taken into account in the scope of this study.

BCC and FCC metals can be studied with this model. In FCC metals, 12 independent slip systems are assumed potentially active, i.e. the slip planes $\{111\}$ and the slip directions <110>. In BCC metals, there are 24 independent slip systems, the slip planes $\{110\}$ and $\{112\}$ and the slip directions $<111>$. The local incremental elastic-plastic constitutive law is defined by means of the tangent modulus $\mathbf{l}$ relating the nominal stress rate $\dot{\mathbf{n}}$ to the velocity gradient $\mathbf{g}$ :

$\dot{\mathbf{n}}=\mathbf{l}: \mathbf{g}$

This velocity gradient is classically decomposed into a symmetric part d corresponding to the total strain rate and a skew-symmetric one $\mathbf{w}$ denoting the total rotation rate (see references [7-10] as well as $[13,14]$ ):

$\mathbf{g}=\mathbf{d}+\mathbf{w}$

These two parts can be further divided into an elastic part with the superscript ${ }^{e}$ and a plastic one with the superscript ${ }^{p}$. The plastic parts (plastic strain rate and plastic spin) are related to the slip rates $\dot{\gamma}^{g}$ by:

$$
\begin{aligned}
& \mathbf{d}^{p}=\mathbf{d}-\mathbf{d}^{e}=\sum_{g} \mathbf{R}^{g} \dot{\gamma}^{g} \\
& \mathbf{w}^{p}=\mathbf{w}-\mathbf{w}^{e}=\sum_{g} \mathbf{S}^{g} \dot{\gamma}^{g}
\end{aligned}
$$

where $\mathbf{R}^{g}$ and $\mathbf{S}^{g}$ denote, respectively, the wellknown symmetric and skew-symmetric parts of the Schmid tensor associated with a given slip system $g$.

For a plastic-type behavior obeying the Schmid law, the definition of the plastic threshold or critical shear stress leads to the following plastic flow rule:

$$
\left\{\begin{array}{l}
\tau^{g}<\tau_{c}^{g} \Rightarrow \dot{\gamma}^{g}=0 \\
\tau^{g}=\tau_{c}^{g} \text { and } \dot{\tau}^{g}<\dot{\tau}_{c}^{g} \Rightarrow \dot{\gamma}^{g}=0 \\
\tau^{g}=\tau_{c}^{g} \text { and } \dot{\tau}^{g}=\dot{\tau}_{c}^{g} \Rightarrow \dot{\gamma}^{g} \geq 0
\end{array}\right.
$$

where $\tau^{g}$ and $\tau_{c}^{g}$ are, respectively, the resolved shear stress acting on the slip system $g$ and the corresponding critical shear stress.

In elastoplasticity, the concept of potentially active $\left(\tau^{g}=\tau_{c}^{g}\right)$ and effectively active $\left(\tau^{g}=\tau_{c}^{g}\right.$ and $\dot{\tau}^{g}=\dot{\tau}_{c}^{g}$ ) slip systems is usually introduced. Within the potentially active slip systems, several subsets of active systems may exist. Thus, it is necessary to find a criterion for selecting the set of active slip systems. A new approach is proposed here in order to avoid combination analyses and to significantly reduce computing time [11,12]. Relation (4) is then replaced with a rate-independent regularization technique $\dot{\gamma}^{g}=k^{g} \dot{\tau}^{g}$, i.e. without introducing rate-sensitivity. Among several possible regularization functions, the following form is adopted for a given slip system $g$ :

$$
k^{g}=\frac{1}{H^{g}} \frac{1}{2}\left(1+\tanh \left(k_{0} \tau^{g}\right)\right) \frac{1}{2}\left(1+\tanh \left(k_{1}\left(\frac{\tau^{g}}{\tau_{c}^{g}}-1\right)\right)\right) \frac{1}{2}\left(1+\tanh \left(k_{2} i^{g}\right)\right)
$$

where $H^{g g}$ is the self-hardening term and 'tanh' stands for the hyperbolic tangent function. Eq. (5) aims at modeling at best a threshold (smooth approximation to the discontinuous step function) without having the numerical problems inherent to the use of sharp functions, and combined with $\dot{\gamma}^{g}=k^{g} \dot{\tau}^{g}$ provides a regular form of the Schmid law, see Eq. (4).

According to the large strain single crystal modeling given in $[13,14]$, Hooke's law is written as:

$$
\hat{\sigma}=\mathbf{C}: \mathbf{d}^{e}-\sigma \operatorname{tr}(\mathbf{d})
$$

where $\mathbf{C}$ is the fourth-order elasticity tensor, $\operatorname{tr}(\cdot)$ denotes the trace operator, $\sigma$ is the Cauchy stress and $\hat{\sigma}$ is the lattice co-rotational derivative given by:

$\hat{\sigma}=\dot{\sigma}+\sigma \cdot \mathbf{w}^{e}-\mathbf{w}^{e} \cdot \sigma$

After mathematical development of the consistency condition, the slip rate expression can be written as:

$\dot{\gamma}^{g}=M^{g h}\left\{k^{h} \mathbf{R}^{h}:(\mathbf{C}-\boldsymbol{\sigma} \otimes \mathbf{1}): \mathbf{d}\right\}$

where 1 stands for the second-order identity tensor, $M^{g h}=\left(\delta^{h g}+k^{h} \mathbf{R}^{h}: \mathbf{C}: \mathbf{R}^{g}\right)^{-1}$ obtained by inverting a matrix in which $\delta^{\text {hg }}$ denotes the identity matrix.

The relation between Cauchy's co-rotational derivative and the nominal stress rate is given by:

$\hat{\sigma}=\dot{\mathbf{n}}+\mathbf{w}^{p} \cdot \sigma-\sigma \cdot \mathbf{w}^{p}-\sigma \operatorname{tr}(\mathbf{d})+\mathbf{d} \cdot \sigma+\sigma \cdot \mathbf{w}$

Combining the previous equations, the single crystal incremental constitutive law, Eq. (1), is obtained:

$$
\begin{aligned}
& l_{i j k l}=\left[C_{i j k l}-\frac{1}{2}\left(\delta_{i k} \sigma_{l j}+\delta_{i l} \sigma_{k j}\right)-\frac{1}{2}\left(\sigma_{i k} \delta_{l j}-\sigma_{i l} \delta_{j k}\right)\right]- \\
& {\left[\left(C_{i j p q} R_{p q}^{g}+S_{i p}^{g} \sigma_{p j}-\sigma_{i p} S_{p j}^{g}\right) M^{g h} k^{h} R_{m n}^{h}\left(C_{m n k l}-\sigma_{m n} \delta_{k l}\right)\right]}
\end{aligned}
$$

This tangent modulus is composed of elastic and plastic parts, where several convective terms appear due to the large strain framework.

It is now necessary to determine the hardening law for the single crystal, i.e. the evolution of critical shear stress rate $\dot{\tau}_{c}$ with slip rate $\dot{\gamma}$ for the whole system. By introducing the hardening matrix $H^{g h}$, the evolution law of the critical shear stress with respect to slip rates can be expressed as:

$$
\dot{\tau}_{c}^{g}=\sum_{h=1}^{n s l i p} H^{g h} \dot{\gamma}^{h}
$$

Interactions of mobile dislocations with lattice and pinned obstacles are the main physical mechanisms of hardening. During plastic strain, dislocations are first created, stored and then annihilated when their densities become large enough. These mechanisms are taken into account in the following evolution law of dislocation density on the slip system $g$ :

$\dot{\rho}^{g}=\frac{1}{b}\left(\frac{1}{L^{g}}-2 y_{c} \rho^{g}\right) \dot{\gamma}^{g}$

where $b$ is the magnitude of the Burgers vector, $y_{c}$ is the critical annihilation distance of dislocations and $L^{g}$ is the mean free path of dislocations on the slip system $g$ expressed by: 


$$
\frac{1}{L^{g}}=\frac{1}{D}+\frac{\sqrt{\sum_{h=1, h \neq g}^{n s i p} \rho^{h}}}{g_{0}}
$$

where $D$ is the average grain size and $g_{0}$ is a parameter related to the dislocation structure.

The relationship between critical shear stress and dislocation densities has been extended to the multislip case by Franciosi [15] as:

$\tau_{c}^{g}=\tau_{0}+\alpha \mu b \sqrt{\sum_{h=1}^{n s l i p} a^{g h} \rho^{h}}$

where $\tau_{0}$ is the critical shear stress for a perfect crystal, $\alpha$ is a constant related to the stability of dislocation configurations, $\mu$ is the shear modulus, and $a^{g h}$ is the anisotropy interaction matrix $[15,16]$. By differentiating Eq. (14) and combining with (12), the hardening matrix can be expressed as follows:

$$
H^{g h}=\frac{\alpha \mu}{2 \sqrt{\sum_{k=1}^{n s l i p} a^{g k} \rho^{k}}} a^{g h}\left(\frac{1}{L^{h}}-2 y_{c} \rho^{h}\right)
$$

\section{Self-consistent model}

To derive the overall polycrystal behavior from knowledge of the behavior of individual grains, a selfconsistent scheme is used. Only the main lines of this classical approach are presented here, all the scheme details are developed in $[10,11]$ as well as in $[13,14]$.

The macroscopic behavior law linking the macroscopic fields - nominal stress rate $\dot{\mathbf{N}}$ and velocity gradient $\mathbf{G}$ - by means of the yet-unknown macroscopic tangent modulus $\mathbf{L}$ has the same incremental form as that of the single crystal (Eq. (1)):

$\dot{\mathbf{N}}=\mathbf{L}: \mathbf{G}$

Those macroscopic fields (i.e. velocity gradient and nominal stress rate) are defined as the volume averages of their microscopic counterparts (see [13]):

$\mathbf{G}=\frac{1}{V} \int_{V} \mathbf{g} d V, \quad \dot{\mathbf{N}}=\frac{1}{V} \int_{V} \dot{\mathbf{n}} d V$

In the self-consistent scale transition scheme, fourthorder concentration tensors, linking macroscopic tensor fields to their microscopic counterparts, are commonly introduced to solve the averaging problem:

$$
\mathbf{g}(\mathbf{x})=\mathbf{A}(\mathbf{x}): \mathbf{G}, \quad \dot{\mathbf{n}}=\mathbf{B}(\mathbf{x}): \dot{\mathbf{N}}
$$

By combining the local behavior law, Eq. (1), with Eqs. (16), (17) and (18), the macroscopic tangent modulus $\mathbf{L}$ can be written in a systematic manner:

$$
\mathbf{L}=\overline{\mathbf{l}(\mathbf{x}): \mathbf{A}(\mathbf{x})}
$$

It is also assumed that the material is a polycrystal constituted of ellipsoidal grains of different crystallographic orientations, and that the behavior and mechanical fields are homogeneous grain by grain. For further derivation, an indicator function $\theta^{I}$, for each grain $I$ of volume $V^{I}$, is defined as:
$\left\{\begin{array}{lll}\theta^{I}(\mathbf{x})=1 & \text { if } & \mathbf{x} \in V^{I} \\ \theta^{I}(\mathbf{x})=0 & \text { if } & \mathbf{x} \notin V^{I}\end{array}\right.$

The local fields can then be expressed in the form:

$$
\mathbf{g}(\mathbf{x})=\sum_{I=1}^{N g} \mathbf{g}^{I} \theta^{I}(\mathbf{x}), \quad \mathbf{l}(\mathbf{x})=\sum_{I=1}^{N g} \mathbf{l}^{I} \theta^{I}(\mathbf{x})
$$

where $\mathbf{g}^{I}$ (respectively $\mathbf{l}^{I}$ ) is the volume average of the velocity gradient (respectively tangent modulus) for the grain $I$, while $N g$ is the number of grains that make up the considered polycrystalline aggregate.

By using Green's tensor, it can be demonstrated, after some elaborate derivations, that the concentration tensor $\mathbf{A}^{I}$ related to grain $I$ is given by:

$$
\mathbf{A}^{I}=\left(\mathbf{I}-\mathbf{T}^{I I}:\left(\mathbf{l}^{I}-\mathbf{L}\right)\right)^{-1}:{\overline{\left(\mathbf{I}-\mathbf{T}^{I I}:\left(\mathbf{l}^{I}-\mathbf{L}\right)\right)^{-1}}}^{-1}
$$

where $\mathbf{T}^{I I}$ is the interaction tensor for grain $I$, related to Eshelby's tensor for an ellipsoidal inhomogeneity. In the case of a polycrystalline aggregate comprising $N g$ grains with their respective volume fraction $f^{I}$, the 1-site self-consistent expression corresponding to the self-consistent scheme in the sense of Hill [17] can be finally obtained as follows:

$$
\mathbf{L}=\sum_{I=1}^{N g} f^{I} \mathbf{l}^{I}: \mathbf{A}^{I}
$$

\section{Ductility loss criterion}

The so-called Rudnicki-Rice criterion [5,6] corresponds to a discontinuous bifurcation phenomenon associated with admissible jumps for strain rates across a shear band as illustrated in Fig. 1.

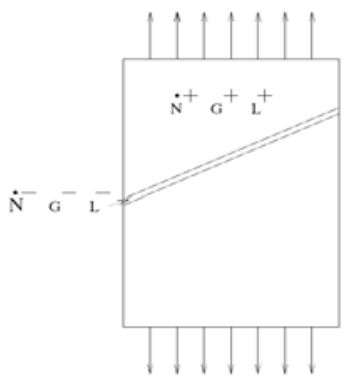

Fig. 1. Localization of the deformation along a shear band.

Since field equations have to be satisfied, the kinematic condition for the strain rate jump is:

$$
[\mathbf{G}]=\mathbf{G}^{+}-\mathbf{G}^{-}=\kappa \otimes v
$$

where $\boldsymbol{\kappa}$ is the jump amplitude and $\boldsymbol{v}$ is the unit vector, normal to the shear band. On the other hand, the continuity of the stress rate vector has to be verified for the forces along the interface created by the localization band, which can be expressed as:

$\left[\dot{\mathbf{N}}^{T}\right] \cdot \mathbf{v}=\mathbf{0}$

At the onset of the bifurcation, it can be considered that the tangent modulus is the same on each part of 
the band. By using the constitutive law (16) together with Eqs. (24-25), the following condition is derived:

$(v \cdot \mathbf{L} \cdot v) \cdot \kappa=\mathbf{0}$

From Eq. (26), two families of solutions can be extracted. The first possibility is when all of the $\kappa \mathrm{s}$ are zero and thus the magnitude of the jumps is zero, in which case there is no bifurcation. The second possibility corresponds to the existence of at least one non-zero $\kappa$, in which case the system of Eq. (26) is undetermined (i.e. it has an infinite number of solutions) leading to the following necessary condition:

$$
\operatorname{det}(\mathbf{v} \cdot \mathbf{L} \cdot \mathbf{v})=0
$$

Each direction of space $v$ that verifies Eq. (27) corresponds to a localization band, in which the amplitude of the jump cannot be calculated directly.

It is worth noting that no additional parameters have been employed here, this criterion associated with the loss of ellipticity of the boundary value problem being intrinsic. This represents one of the strong advantages over the M-K classical approach.

\section{Identification of material parameters}

In this section, the results obtained with the proposed model are compared to experimental ones. Several sequential rheological tests have been performed for a single-phase ferritic steel. A necessary preliminary step has been to identify the four following material parameters for this steel:

- The initial critical shear stress $\tau_{0}$ is assumed to be the same for all slip systems. In the case of a uniaxial tensile test and for a single-phase steel, its value is approximately one half of the elastic limit.

- The parameter $g_{0}$ is associated with the mean free path of dislocations. Its influence is found to be strong during the first stages of deformation.

- The critical annihilation distance $y_{c}$ has a significant effect mainly when deformation becomes large enough.

- The average grain size $D$ can be easily identified using optical micrography.

These parameters are identified by means of two stress-strain curves: a uniaxial tensile test and a Bauschinger shear test until $10 \%$ of logarithmic strain for the first path. The identified parameters for this steel are reported in Table 1 below.

Table 1

Identified parameters for the single-phase steel

\begin{tabular}{ccccc}
\hline$\tau_{0}[\mathrm{MPa}]$ & $\rho_{0}\left[\mathrm{~m}^{-2}\right]$ & $g_{0}$ & $y_{c}[\mathrm{~m}]$ & $D[\mathrm{~m}]$ \\
\hline 45 & $1.10^{-9}$ & 90 & $3.2510^{-9}$ & $20.10^{-6}$ \\
\hline
\end{tabular}

\section{Numerical results and comparison with experiments - macroscopic behavior}

In Fig. 2 below, comparisons between simulations and experiments for the previous steel are depicted.
These are monotonic tests (uniaxial tension and simple shear) and two-stage sequential tests (Bauschinger tests at three different pre-strains and an orthogonal test: a uniaxial tension followed by a simple shear).

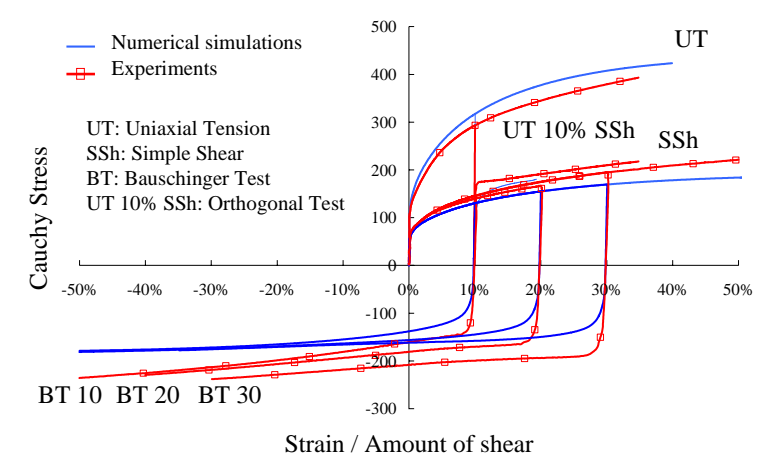

Fig. 2. Comparison between rheological test simulations and experiments for a single-phase steel. Bauschinger Tests are performed at $10 \%, 20 \%$ and $30 \%$ of shear strain; UT $10 \%$ SSh stands for Uniaxial Tension until $10 \%$ of logarithmic strain followed by Simple Shear.

It is shown that the proposed model is able to reproduce the elastic-plastic behavior of single phase polycrystalline materials and that numerical results are in close agreement with the experimental ones. However, some differences exist especially during sequential loading paths (Bauschinger tests and orthogonal test). As can be observed in Fig. 2 with the Bauschinger tests, these gaps increase as pre-strain gets larger. This can be explained by the fact that the spatial rearrangement of dislocation cells is not taken into account in the present model. Experimental evidence of such a rearrangement can be found easily in the literature for IF-steels [18]. In the present model, the internal variable acting at this scale is the mean dislocation density for a slip system. At the onset of the second loading path, the structure of the dislocation cells is disintegrated and another one, depending on the second loading path, is created [19], which may explain the observed differences. One idea, in order to reduce this gap, would consist of introducing kinematic hardening at the slip system level (the kinematic hardening in the present model is only inter-granular and not intra-granular due to the self-consistent scheme).

\section{Numerical results and comparison with experiments - ductility loss predictions}

In Fig. 3, comparisons between simulations and ArcelorMittal's FLD for the previous steel are depicted. In order to obtain a representative curve allowing comparison with the reference FLD, a polynomial interpolation has been used. As can be observed, the computed FLDs are close to the reference FLDs provided by ArcelorMittal. The positive domain seems to be overestimated, but the introduction of a damage model should very likely improve the results in this part. Likewise, it can also be seen that some localization points, given by the 
model, exhibit a certain dispersion in the expansion domain. This is mainly due to statistics related to grain to grain heterogeneities. Further research is currently being undertaken to better understand this phenomenon.

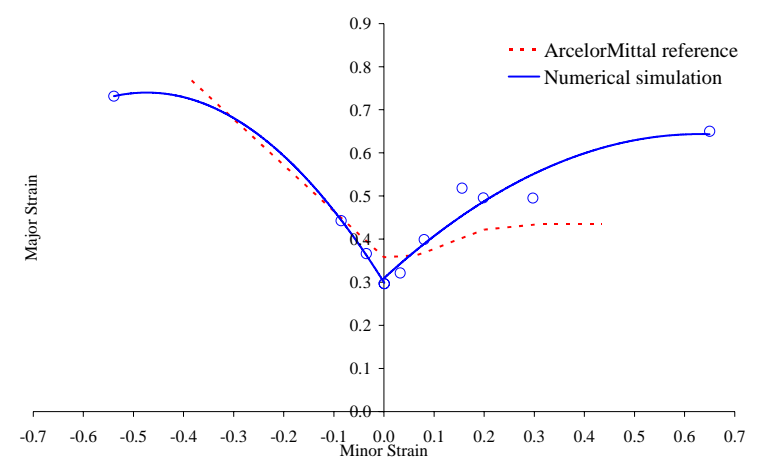

Fig. 3. Loss of ellipticity prediction: comparison between linear, computed FLD and reference FLD for a single-phase steel.

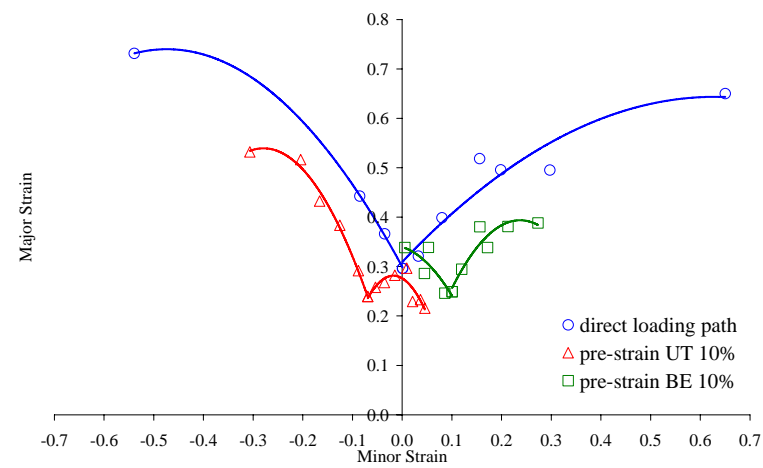

Fig. 4. Loss of ellipticity prediction: comparison between linear and complex FLDs for a single-phase steel. UT 10\% stands for $10 \%$ of Uniaxial Tension logarithmic pre-strain, BE $10 \%$ for $10 \%$ of Biaxial Expansion logarithmic pre-strain.

In Fig. 4 above, numerical FLDs are plotted with two different pre-strains for this single-phase steel. Experimental observations on different steels have shown that if the pre-strain is under biaxial expansion, the FLD shifts to the right and down with respect to the direct path FLD, whereas it shifts to the left and up if the pre-strain is in uniaxial tension [20]. These results are partly reproduced by the model. It is clearly observed in Fig. 4 that the biaxial expansion pre-strain shifts the curve to the right and the uniaxial tension shifts the curve to the left. However, it can be noticed that the two-stage FLD obtained after a uniaxial tension pre-strain tends to be slightly underestimated.

\section{Shear band orientation}

In this section, the three-dimensional effect of necking analysis will be emphasized. Indeed, as mentioned in the introduction, our approach based on Rice's bifurcation criterion allows a fully threedimensional analysis of shear band formation. In contrast to most of the previously published contributions, based on plane-stress assumptions, the proposed model is able to give information regarding the through-thickness orientation of the localized band. As shown in Fig. 5, the band orientation can be defined thanks to two angles: the inclination of the band $\theta_{1}$ with respect to rolling direction $\mathrm{RD}$ in the sheet plane (RD, TD), and its inclination $\theta_{2}$ with respect to rolling direction $\mathrm{RD}$ in the thickness plane (RD, ND). Two different loadings - uniaxial tension and plane tension - have been considered for this single-phase steel and the results of orientation analysis are reported in Table 2.

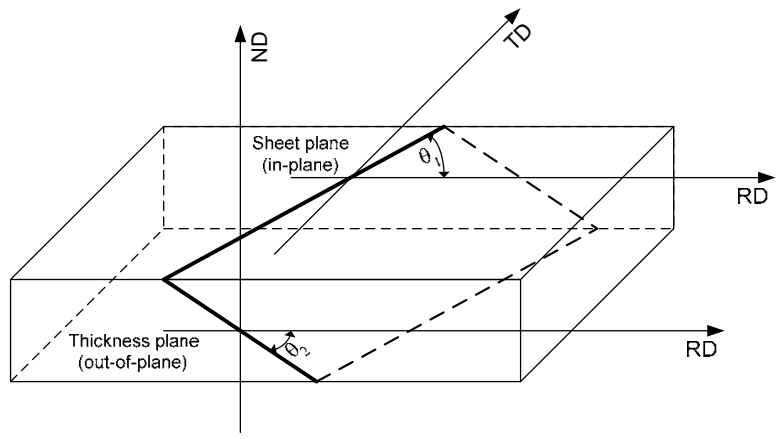

Fig. 5. In-plane and out-of-plane orientation of shear band.

For the uniaxial tensile test, the in-plane band inclination with respect to rolling direction is found to be $50^{\circ}$. This result is close to the value of $54^{\circ}$ generally observed in experiments. This latter in-plane orientation can also be recovered by means of Hill's criterion [21], which gives a direct relation between the angle and the principal strain ratio. This value for the in-plane orientation is also found in [22] using both an analytical formulation and an elastic-plastic model coupled with damage implemented into a finite element code. Another recent contribution [23], in which Rice's bifurcation criterion has been used in conjunction with an advanced elastic-plastic model coupled with damage, also provides the value of $54^{\circ}$ for the in-plane orientation. Detailed investigations in this regard [24] reveal that the orientation of shear bands can depend on the texture and anisotropy. Texture effects may explain the slight difference between the above-mentioned results. With regard to thickness, the proposed model predicts a band perpendicular to the sheet plane, which is in agreement with the investigations conducted in [23].

Table 2

In-plane and out-of-plane orientation of the localization band for two different loading modes

\begin{tabular}{cccc}
\hline & Steel & \multicolumn{2}{c}{ Single Phase } \\
\cline { 2 - 4 } & Orientation & $\theta_{1}$ & $\theta_{2}$ \\
\hline \multirow{2}{*}{ Loading mode } & Uniaxial Tension & $50^{\circ}$ & $90^{\circ}$ \\
& Plane Tension & $90^{\circ}$ & $46^{\circ}$ \\
\hline
\end{tabular}

For the plane tensile test, the model predicts a band perpendicular to the rolling direction (i.e. the in-plane orientation with respect to rolling direction is $\theta_{1}=90^{\circ}$ ), which is in agreement with many experiments and also with recently published contributions $[23,24]$. For the 
out-of-plane inclination of the band, the model gives the value of $46^{\circ}$ with respect to rolling direction; which is also consistent with previous analyses [23].

\section{Conclusions}

In this paper, a multiscale model has been combined with Rice's ellipticity loss criterion in order to analyze the formability of steels in sheet forming processes. Numerical FLDs have been plotted and compared to reference ones. In this regard, the macroscopic behavior law has been accurately modelled in order to take into account the most important softening mechanisms so that the effects of complex loading paths are at least partially captured. This has been made possible thanks to the use of a robust self-consistent scale-transition scheme within finite strain in conjunction with a new regularization technique for the selection of active slip systems, which significantly improves computational efficiency.

Although less popular than other approaches, more commonly used in sheet metal forming processes (e.g. M-K analyses, Maximum Force Criteria), the adopted Rice's criterion, based on loss of ellipticity, has proven to be able to determine FLDs, which have a very similar shape to experimental FLDs and reproduce qualitatively the effects of complex loading paths.

This resulting theoretical and numerical tool allows the ductility prediction of new materials from the early stage of the design of new grades of steel and thus provides a powerful and useful tool for steelmakers. Its main interest is to allow comparisons in terms of formability of those materials and to determine the impact of microstructural effects on the ductility. Therefore, it can be advantageously used to optimize the ductility of new steels or to design materials with desired formability and mechanical properties in-use.

Contrary to more phenomenological approaches, it is worthwhile to notice that the proposed model does not need any additional parameter to capture the occurrence of strain localization. It also allows threedimensional effects of shear bands to be reproduced.

\section{Acknowledgements}

The authors are grateful to ArcelorMittal for providing them with FLD experimental data allowing interesting comparisons and for its financial support to this research.

\section{References}

[1] S.P. Keeler, Determination of forming limits in automotive stampings, Sheet Metal Indust. 42 (1965) 683-691.

[2] G.M. Goodwin, Application of strain analysis to sheet metal forming problems in the press shop, SAE Paper No. 680093 (1968).

[3] Z. Marciniak, K. Kuczynski, Limit strains in the processes of stretch-forming sheet metal, International Journal of Mechanical Sciences 9 (1967) 609-620.

[4] J.W. Hutchinson, K.W. Neale, Mechanics of Sheet Metal Forming, Plenum Press, New York, 1978.
[5] J.W. Rudnicki, J.R. Rice, Conditions for the localization of deformation in pressure-sensitive dilatant materials, Journal of the Mechanics and Physics of Solids 23 (1975) 371-394.

[6] J.R. Rice, The localization of plastic deformation, in: 14th International Congress of Theoretical and Applied Mechanics, 1976, pp. 207-220.

[7] R.J. Asaro, Crystal plasticity, Journal of Applied Mechanics 50 (1983) 921-934.

[8] D. Peirce, Shear band bifurcations in ductile single crystals, Journal of the Mechanics and Physics of Solids 31 (2) (1983) 133-153.

[9] D. Pierce, R.J. Asaro, A. Needleman, Material rate dependence and localized deformation in crystalline solids, Acta Metallurgica 31 (12) (1983) 1951-1976.

[10] T. Iwakuma, S. Nemat-Nasser, Finite elastic-plastic deformation of polycrystalline metals and composites, Proc. R. Soc. Lond. A 394 (1984) 87-119.

[11] J.P. Lorrain, Critère de ductilité basé sur la perte d'ellipticité du module tangent élastoplastique déduit d'un modèle autocohérent, PhD. Thesis, ENSAM Metz, France, 2005.

[12] J.P. Lorrain, T. Ben Zineb, F. Abed-Meraim, M. Berveiller, Ductility loss modelling for BCC single crystals, Int. J. Forming Proc. 8 (2-3) (2005) 135-158.

[13] P. Lipinski, M. Berveiller, Elastoplasticity of microinhomogeneous metals at large strains, International Journal of Plasticity 5 (1989) 149-172.

[14] P. Lipinski, M. Berveiller, E. Reubrez, J. Morreale, Transition theories of elastic-plastic deformation of metallic polycrystals, Archive of Applied Mechanics 65 (1995) 291-311.

[15] P. Franciosi, Etude théorique et expérimentale du comportement élastoplastique de monocristaux métalliques se déformant par glissement: Modélisation pour un chargement complexe quasi-statique, $\mathrm{PhD}$. Thesis, Paris North University, France, 1984.

[16] T. Hoc, Etudes expérimentales et numériques de la localisation de la déformation dans les aciers doux, PhD. Thesis, Ecole Centrale de Paris, France, 1999.

[17] R. Hill, Continuum micro-mechanics of elastoplastic polycrystals, Journal of the Mechanics and Physics of Solids 13 (1965) 89-101.

[18] E.V. Nesterova, B. Bacroix, C. Teodosiu, Microstructure and texture evolution under strain path changes in low-carbon interstitial-free steel, Metallurgical and Materials Transactions A 32A (2001) 2527-2538.

[19] B. Peeters, Multiscale modelling of the induced plastic anisotropy in IF steel during sheet forming, $\mathrm{PhD}$. Thesis, Katholieke Universiteit Leuven, Belgium, 2002.

[20] A. Haddad, Contribution à la détermination des courbes limites de formage en contraintes et en déformations à partir de la théorie 3G, PhD. Thesis, Université de Savoie, France, 1997.

[21] R. Hill, The Mathematical Theory of Plasticity, Oxford University Press, Oxford, 1950.

[22] I. Doghri, R. Billardon, Investigation of localization due to damage in elasto-plastic materials, Mechanics of Materials 19 (1995) 129-149.

[23] B. Haddag, F. Abed-Meraim, T. Balan, Strain localization analysis using large deformation anisotropic elastoplastic model coupled with damage, In: $3^{\text {rd }}$ ECCM Conference, Lisbon, 2006.

[24] M. Kuroda, V. Tvergaard, Effects of texture on shear band formation in plane strain tension/compression and bending, International J. Plasticity 23 (2007) 244-272. 